\title{
Measuring Waves and Currents with an Upward-Looking ADCP
}

\author{
Eugene A. Terray \\ Department of Applied Ocean Physics and Engineering \\ Woods Hole Oceanographic Institution \\ Woods Hole, MA 02543 \\ eterray@whoi.edu \\ Blair H. Brumley and Brandon Strong \\ RD Instruments, Inc. \\ San Diego, CA
}

\begin{abstract}
Routine monitoring of waves and currents in the nearshore region is of great interest both scientifically and to the general public because of their role in coastline erosion and their impact on recreational activities. Historically, the technology for measuring these quantitites has been distinct, requiring separate instrumentation for each. In this contribution we show that it is possible in shallow water to estimate both wave height and direction from a conventional bottom-mounted, upward-looking Acoustic Doppler Current Profiler. Height and direction spectra compare well with a co-located array of pressure gages.
\end{abstract}

\section{Motivation AND APPROACH}

We have investigated the possibility of measuring both waves and currents from a single bottom-mounted ADCP. Because most existing ADCPs utilize the "Janus" beam geometry (shown in Fig. 1a), and do not incorporate a pressure sensor, we were strongly motivated to focus on wave estimation techniques that would work acceptably with this configuration. Our goal was to obtain performance that is at least comparable to that of the wave direction sensors, such as pressure/velocity (PUV) triplets or directional wave buoys, that have traditionally been used for this purpose. However, we have also investigated the performance of the more specialized "Bugeye" $3+1$ configuration shown in Fig. 1b. This consists of a single vertical plus three inclined beams (oriented at 120 degree increments in azimuth) and uses the same electronics as a Janus-configured instrument. The $3+1$ beam geometry permits current profiling, and also has several advantages for measuring waves that we will discuss later.

ADCPs intended for current profiling employ acoustic beams inclined at an angle with respect to the vertical (typically by 20-30 degrees). The sonar measures the instantaneous velocity component projected along each beam, averaged over a range cell. Since the "mean" current is typically horizontally uniform over the beams, its components can be recovered as linear combinations of the measured along-beam velocities. The situation regarding waves is more complicated since at any instant the wave velocities vary spatially across the array. As a result, except for very long waves that remain coherent during their passage

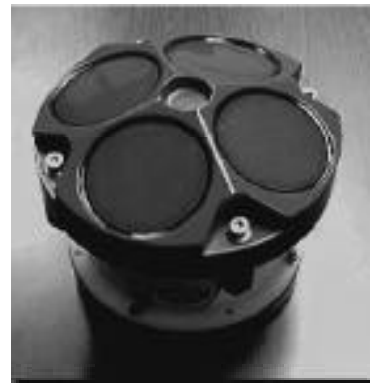

Fig. 1a. Standard "Janus" ADCP

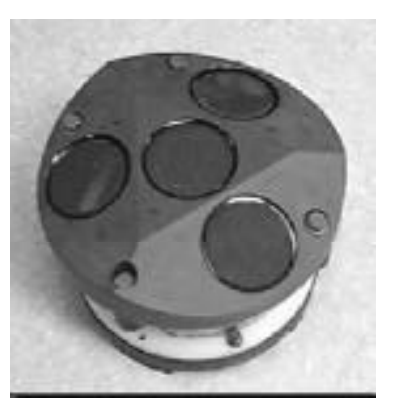

Fig. 1b. Vertical beam " $3+1$ " through the array, it is not possible to separate the horizontal and vertical wave velocity components. However, the wave field is statistically stationary in time and homogeneous in space, and therefore cross-spectra between velocities measured at various range cells (either beam to beam or along each beam) contain information about wave direction.

While it is true that the collection of ADCP range cells constitutes an array, it is a somewhat peculiar one. First, the signal-to-noise ratio (SNR) in the measured velocities varies with depth. At a particular frequency this variation is due primarily to the vertical decay of the wave energy, and (to a lesser extent) on the acoustic SNR, which itself is a function of range. Second, the velocity measured by the ADCP is a linear combination of horizontal and vertical wave velocities, with relative weights that depend on both the propagation direction of the waves, and the height of the measurement cell above the bottom. For example, in deep water as the wave direction varies from 0 to 90 degrees, the measured along-beam velocity varies by only $25 \%$. Lastly, because the range cells closest to the surface have the highest velocity SNR, the usable array lag distribution is quite sparse, consisting of long lags from beam to beam, and a second group of shorter lags between range cells along each beam. The exact number of useful lags depends on the details of the wavenumber distribution of the waves and the water depth. It is clear from these considerations that unlike conventional point sensors, the "sensor array" associated with an upward- 
looking ADCP does not have a simple relationship with the low order circular moments of the wave directional distribution, and therefore that recovering wave direction is considerably more complicated in this case. We will discuss this problem more fully in Section V.

\section{OVERVIEW OF FIELD DATA}

\section{A. FRF Deployment}

Data were collected during the spring and summer of 1993 at the Field Research Facility (FRF) of the US Army Engineer Waterways Experiment Station at Duck, North Carolina.

The ADCP was located roughly $1 \mathrm{~km}$ offshore in $8 \mathrm{~m}$ water depth, close to the FRF's pressure gage array which provided comparison observations. The ADCP configuration and setup are summarized in Table 1.

$\begin{aligned} & \text { TABLE } 1 \\ & \text { FRF ADCP CONFIGURATION } \\ & \text { Model: } \text { RDI BroadBand } \\ & \text { Frequency: } 1200 \mathrm{kHz} \\ & \text { Beam angle: } 30^{\circ} \text { relative to vertical } \\ & \text { Transducer: } \text { Convex } \\ & \text { Firmware: } 3.04^{*} \\ & \text { Bin size: } 0.5 \mathrm{~m} \\ & \text { Number of bins: } 12 \\ & \text { Blank: } 3.3 \mathrm{~m} \\ & \text { Mode: } 4 \\ & \text { Sample interval: } 0.7 \mathrm{sec} \\ & \text { Pings per ensemble: } 1 \\ & \text { Heading } 313^{\circ} \text { re Magnetic North } \\ & \text { Magnetic declination } 10^{\circ} \mathrm{W} \text { of True North } \\ & \text { Tilt 1 }+3.9^{\circ} \text { (beam 3-4 axis) } \\ & \text { Tilt } 2-9.4^{\circ} \text { (beam 1-2 axis) } \\ &\end{aligned}$

\section{B. SIO Deployment:}

The largest significant wave heights encountered during the FRF deployment were slightly in excess of $1.3 \mathrm{~m}$. In order to examine the ability of the ADCP to measure large wave orbital velocities, we deployed an ADCP in $11 \mathrm{~m}$ water depth, several hundred meters seaward of the end of the Scripps Institution of Oceanography (SIO) pier in La Jolla, CA. The details of the ADCP configuration are listed in Table 2. We have operated this instrument more or less continuously from April, 1997 to July, 1998. During April, 1998, the instrument described in Table 2 was replaced by a $1200 \mathrm{kHz}$ ADCP configured with the " $3+1$ " beam geometry (Fig. 1b).

\begin{aligned} & \hline TABLE 2 \\ & SIO ADCP CONFIGURATION \\ & Model: RDI WorkHorse \\ & Frequency: $1200 \mathrm{kHz} \\ &$ Beam angle: $20^{\circ}$ relative to vertical \\ & Transducer: Convex \\ & Firmware: $3.04^{*} \\ &$ Bin size: $0.25 \mathrm{~m} \\ &$ Number of bins: 50 \\ & Blank: $2.07 \mathrm{~m} \\ &$ Mode: 1 \\ & Sample interval: $0.5 \mathrm{sec} \\ &$ Pings per ensemble: 1 \\ & Heading $353^{\circ}$ re Magnetic North \\ & Magnetic declination $13^{\circ} \mathrm{E}$ of True North \\ & Tilt 1$-0.06^{\circ}$ (beam 3-4 axis) \\ & Tilt 2$+0.51^{\circ}$ (beam 1-2 axis) \\ & \hline\end{aligned}

\section{DETERMinATION OF WATER DEPTH}

The directional estimation algorithm requires a forward model describing the connection between the measured crossspectra and the frequency-direction distribution of the waves. The latter, an example of which is specified by Equations 3-6 in Section V, requires that the water depth be known. Since most existing ADCPs are not equipped with a pressure sensor, we have explored the idea, originally due to Visbeck and Fischer [9], of using echo-ranging to determine the distance from the ADCP to the surface (we are assuming that the height of the transducers above the bottom is known as part of the installation). The basic idea is illustrated in Fig. 2 for both the Janus and $3+1$ beam geometries. Note that in the latter case, only the vertical beam is usable since its surface return is sufficiently large relative to the slant beams to mask their returns. The range to the surface along each beam is determined by quadratic interpolation using the bin having the maximum echo intensity plus its immediate neighbors at shorter and greater ranges. The error in such a procedure behaves similarly to quantization error, and for wave amplitudes large compared to a range cell the bias error is zero on average. For wave amplitudes comparable to the range cell size, on the other hand, the bias can be significant. In the worst case of peak-picking without interpolation, and calm water at a level halfway between two range bins, the bias is roughly one half a bin.

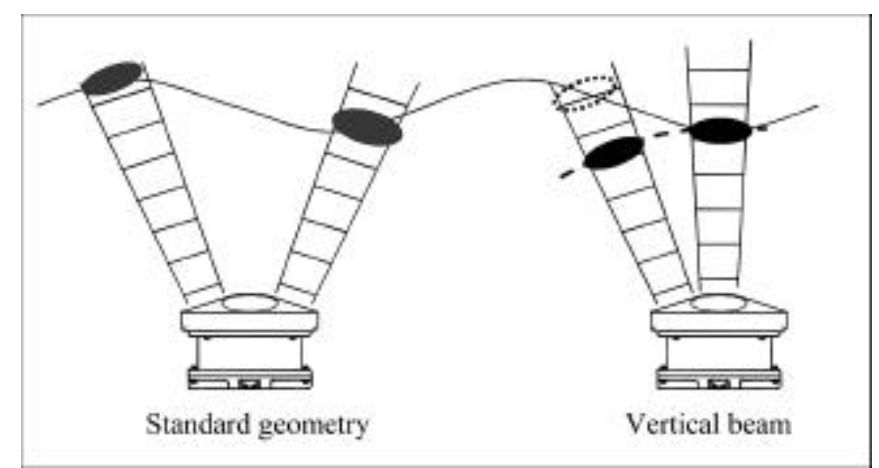

Fig. 2. 
Based on our limited measurements with the $3+1$ geometry and the more extensive measurements reported by Zedel [10] using a vertically-oriented sonar, we conclude that a vertical beam provides a reliable measurement of water depth under almost all wind and wave conditions. Somewhat to our surprise, we found that the conventional slant-beam Janus configuration also works well, except during periods of swell coupled with light winds. Under such smooth surface conditions, the scattering is mostly specular, making it difficult to differentiate the main and sidelobe returns. In the Janus data we have examined to date, such periods have been short relative to a tidal cycle, and easy to identify in the data. Coupling the observations to a tidal model (where the data are used to initialize the model and to control the growth of error) would permit periods of dropout to be interpolated over. We also note that bubbles did not appear to bias the results obtained using either beam geometry. This is probably due to the high acoustic frequency employed here (1200 $\mathrm{kHz}$ ), and the intermittency of intense bubble plumes (associated with breaking waves and Langmuir cells) at a fixed location (note that each beam subtended an area at the surface roughly $0.5 \mathrm{~m}$ in diameter).

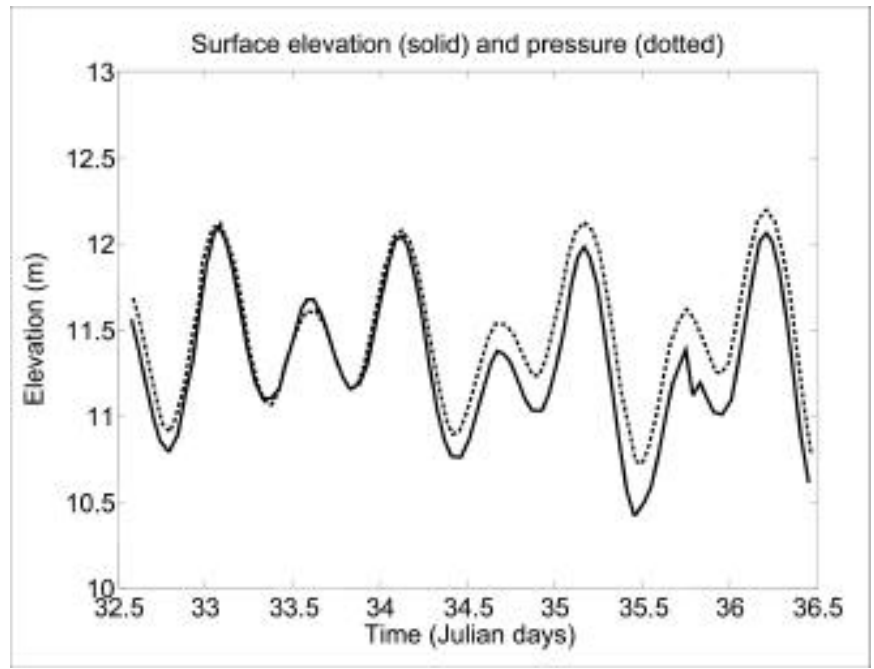

Fig. 3.

An example of the performance over 4 days of a 1200 $\mathrm{kHz}$ Janus ADCP deployed beyond the SIO pier at a depth of $11 \mathrm{~m}$ is shown in Fig. 3, where we compare the surface height obtained by echo-ranging to that computed from a pressure sensor mounted on the ADCP. Although the agreement is generally quite good, there is a small, slowly varying, discrepancy. The source of the disagreement can be seen in Fig. 4 which shows that the difference between the pressure and echo-ranging measurements of water depth correlates closely with the change in barometric pressure recorded on the SIO pier.

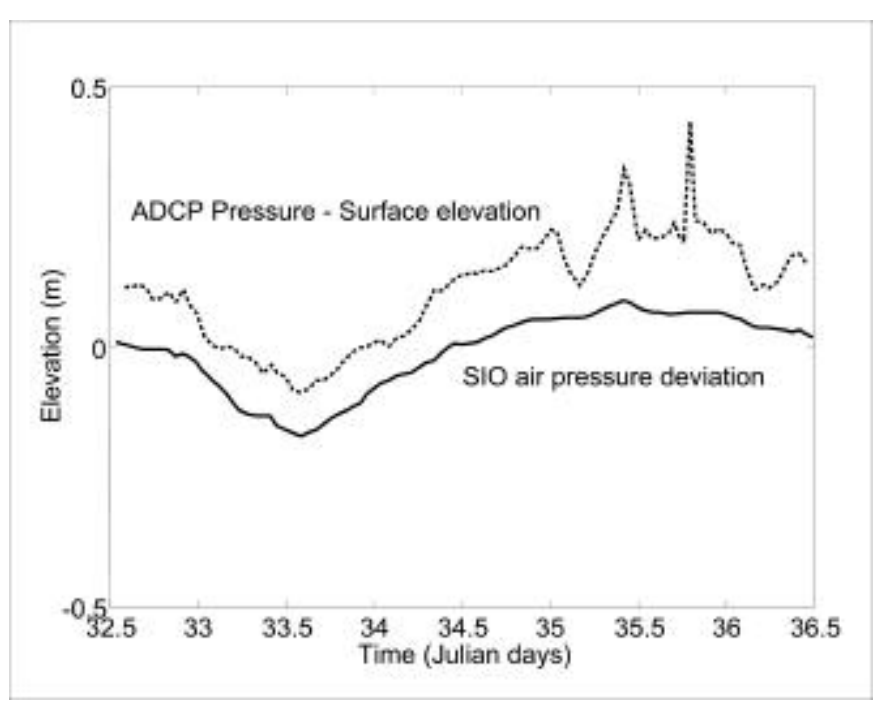

Fig. 4.

\section{MEASURING Wave HeIght}

The scalar wave height spectrum can be estimated in two ways: either from the measured velocity spectra, or by means of the echo-ranging technique. We discuss each of these in turn below.

\section{A. Echo-Ranging}

We have found that the echo-ranging technique described in the preceding section in connection with determining water depth on tidal time scales can in many cases also provide useful measurements of surface elevation in the wave band of frequencies. This is illustrated in Figs. 5 and 6, which show time series of surface elevation from both the " $3+1$ " and Janus configurations, respectively. In Fig. 5 the surface elevation is estimated via echo-ranging. The lower panel in Fig. 6 also shows surface displacement computed from a colocated pressure sensor. The corresponding spectra are shown in Fig.s 7 and 8. In Fig. 8, $S_{e}$ and $S_{p}$ denote the height spectra derived from echo-ranging and the pressure sensor, respectively.

\section{B. Subsurface Velocity}

The vertical wave velocity is non-directional, and its spectrum, $S_{w}$, measured at a height $\mathrm{z}$ above the bottom, is related to the spectrum of wave height, $S_{\eta}$, as

$$
S_{w}(\omega, z)=\left[\omega \frac{\sinh (k z)}{\sinh (k d)}\right]^{2} S_{\eta}(\omega)+S_{N}(\omega)
$$

where $S_{N}$ is the spectrum of the Doppler velocity error [1], and $\mathrm{d}$ is the water depth. Hence for the $3+1$ geometry, the vertical velocity can be used to estimate the wave height spectrum [10]. Estimates of both $\mathrm{S}_{\eta}(\omega)$ and $\mathrm{S}_{\mathrm{N}}(\omega)$ can be 
obtained via a least squares fit to Equation 1, viewed as a function of sensor height $\mathrm{z}$. The results are shown in Fig. 7, where it is seen that the echo-ranging and velocity-derived wave height spectra agree quite well for frequencies below roughly $0.45 \mathrm{~Hz}$.

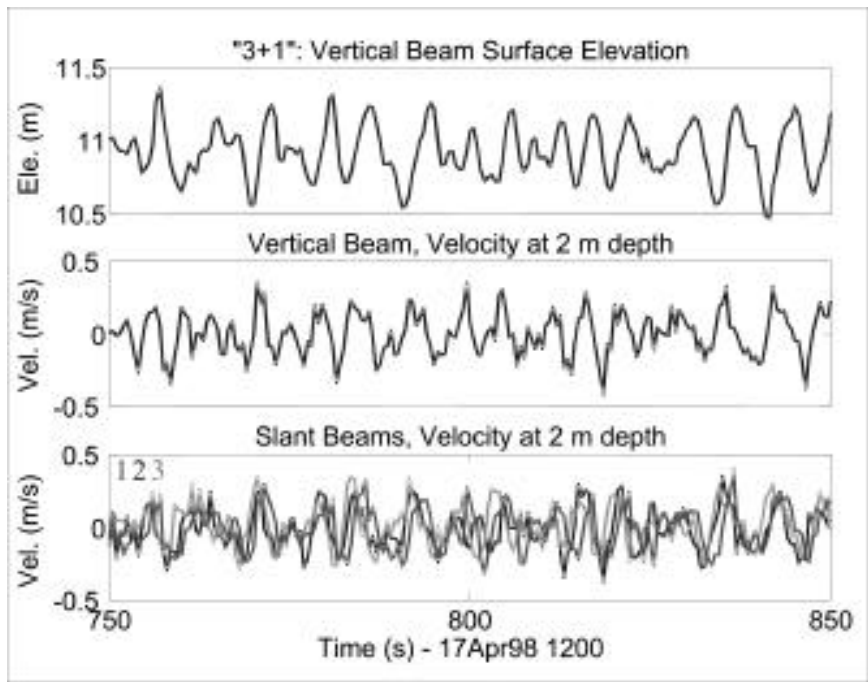

Fig. 5

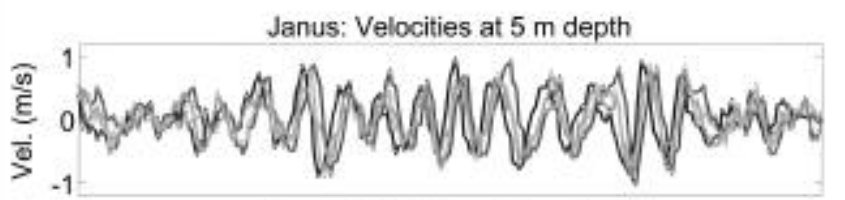

Janus: Velocities at $3 \mathrm{~m}$ depth
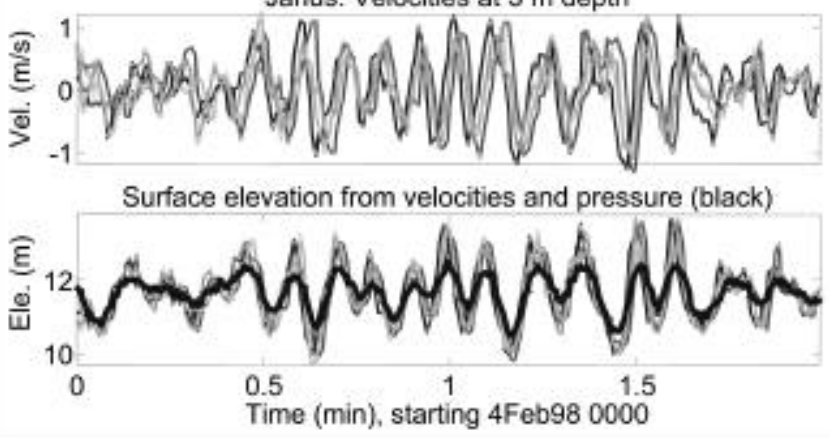

Fig. 6

In the case of a Janus configuration, the linear combination at any depth of the velocity spectra from each of the four range cells is independent of wave direction. Schematically we can write this as

$$
\sum_{n=1}^{4} S_{V}\left(\omega, x_{n}, z\right)=T(\omega, z, d) S_{\eta}(\omega)+S_{N}(\omega)
$$

where $S_{\mathrm{v}}\left(\omega, \mathbf{x}_{\mathrm{n}}, \mathrm{z}\right)$ denotes the spectrum of velocity measured by the $\mathrm{n}^{\text {th }}$ acoustic beam at a height $\mathrm{z}$ above the bottom.

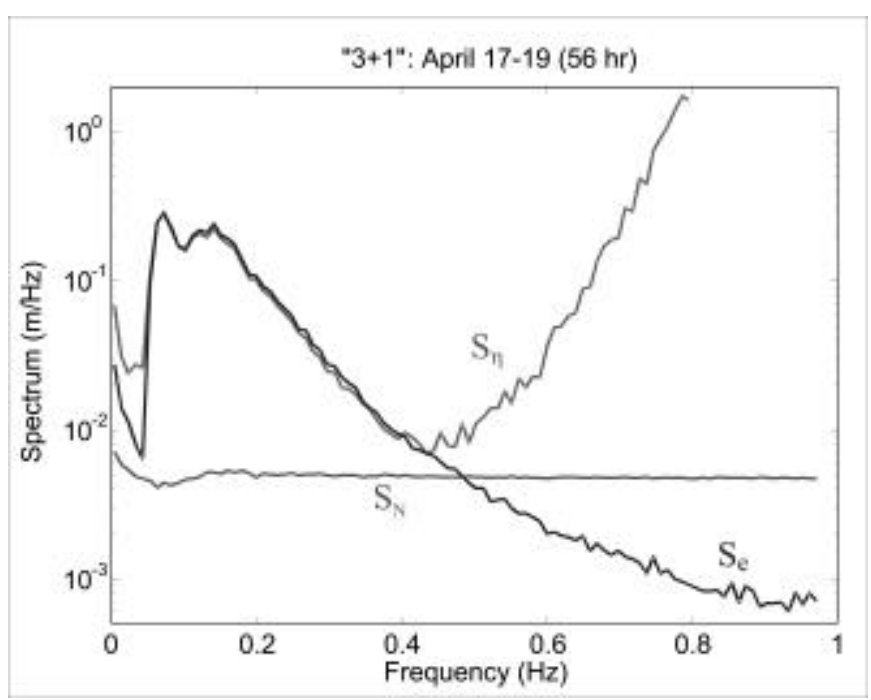

Fig. 7

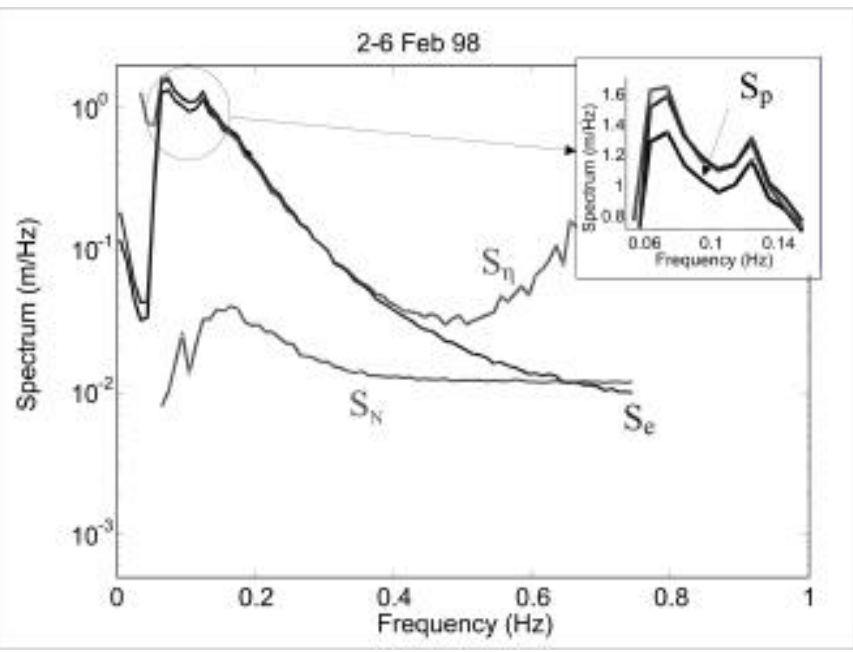

Fig. 8

Although the expression for the transfer function, $T(\omega, \mathrm{z}, \mathrm{d})$ in Equation 2, is more complicated than the form given in Equation 1, estimates of $S_{\eta}(\omega)$ and $S_{N}(\omega)$ can again be recovered using least squares. The results of this procedure for the height and noise spectra are shown in Fig. 8. The insert also shows the height spectrum, denoted by $\mathrm{S}_{\mathrm{p}}$, derived from a pressure sensor co-located with the ADCP. Note that the height spectra estimated from wave velocity and echoranging agree closely for frequencies below roughly $0.35 \mathrm{~Hz}$, whereas both deviate from the pressure-derived spectrum in the region about the peak (this is emphasized in the insert). The reason for this discrepancy is unknown. However, because of the close agreement between the velocity-based and echo-ranging estimates of wave height from the ADCP, we view it as a cautionary note on the difficulties that can be encountered using bottom-mounted pressure sensors to measure wave height. 
Another comparison, obtained at the FRF site, between ADCP velocity- and pressure-derived height spectra (the latter from the FRF pressure gage array) is shown in Fig. 9. The waves in this case were quite small (the significant height was approximately $0.3 \mathrm{~m}$ ), and the observation periods for the ADCP and pressure gage were offset by $1 / 2$ hour. The three solid curves give the height spectrum derived from ADCP velocity measurements at heights of 4,5 and $6 \mathrm{~m}$ above the bottom (the water depth was $8 \mathrm{~m}$ ).

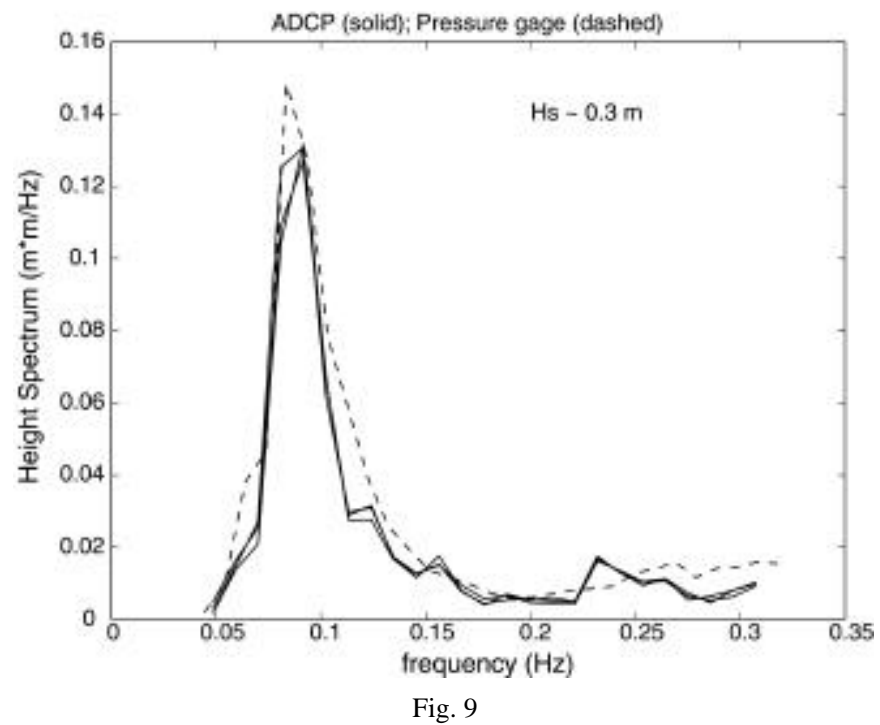

\section{DETERMINING WAVE DiRECTION}

A number of methods have been invented to estimate the direction of multiple arrivals using sparse arrays (see for example, [4]). We use an iterative version of Capon's "Maximum Likelihood Method" [2] known as the IMLM [5, 6]. This technique has the advantage that it does not require that the array be uniformly spaced). Iteration improves the consistency of the MLM estimate, in the sense that the estimated directional spectrum produces (via Equation 3) cross-spectra in closer agreement with the observations. The result is to sharpen the directional resolution, and decrease sidelobes. Like all high resolution direction-of-arrival estimators, the MLM is model-based and requires a relation between the frequency-direction spectrum and the array covariance.

The cross-spectrum $C\left(\mathbf{x}, \mathbf{x}^{\prime} \mid \omega\right)$, between two range cells located at $\mathbf{x}$ and $\mathbf{x}^{\prime}$ (measured relative to the ADCP and the bottom) is related to the wave spectrum as

$\mathrm{C}\left(\mathbf{x}, \mathbf{x}^{\prime} \mid \omega\right)=S(\omega) \int_{-\pi}^{+\pi} d \vartheta \mathrm{H}(\vartheta, \omega, \mathbf{x}) D(\omega, \vartheta) H^{+}\left(\vartheta, \omega, \mathbf{x}^{\prime}\right)$ where $\omega$ denotes angular frequency (radians/second), $S(\omega)$ the wave height spectrum, and $\mathrm{D}(\omega, \vartheta)$ the frequencydirection spectrum (normalized to unit area) $[7,8] . \quad H(\vartheta, \omega, \mathbf{x})$ is the expected velocity at position $\mathbf{x}$ in the presence of a plane wave of unit amplitude and radian frequency $\omega$, propagating in the direction $\vartheta$, and is given by

$$
H(\vartheta, \omega, \mathbf{x})=\mathbf{e}(\mathbf{x}) \cdot \hat{\mathbf{u}}(\omega, \vartheta, z) e^{i \mathbf{k} \cdot \mathbf{x}}
$$

In this equation $\mathbf{e}(\mathbf{x})$ is a unit vector in the direction of the range cell at location $\mathbf{x}$, and $\hat{\mathbf{u}}(\omega, \vartheta, \mathrm{z})$ denotes the Fourier components of the velocity vector of a plane wave having wave number $\mathbf{k}=\mathrm{k}[\cos \vartheta$, $\sin \vartheta]$. We use the result for $\hat{\mathbf{u}}$ given by linear theory

$\hat{\mathbf{u}}(\omega, \vartheta, \mathrm{z})=\omega[\cosh (k z) \cos \vartheta, \cosh (k z) \sin \vartheta,-i \sinh (k z)] / \sinh (k d)$

where $d$ is the water depth. The magnitude of the wave number is assumed to be related to $\omega$ via the linear theory dispersion relation,

$$
\omega^{2}=g k \tanh (k d)
$$

Fig. 10 shows an example of the IMLM frequencydirection spectrum, $S(\omega) \mathrm{D}(\omega, \vartheta)$, obtained from both the ADCP and gage array (the case shown is the same as that in Fig. 11). More comparisons are given in [7].
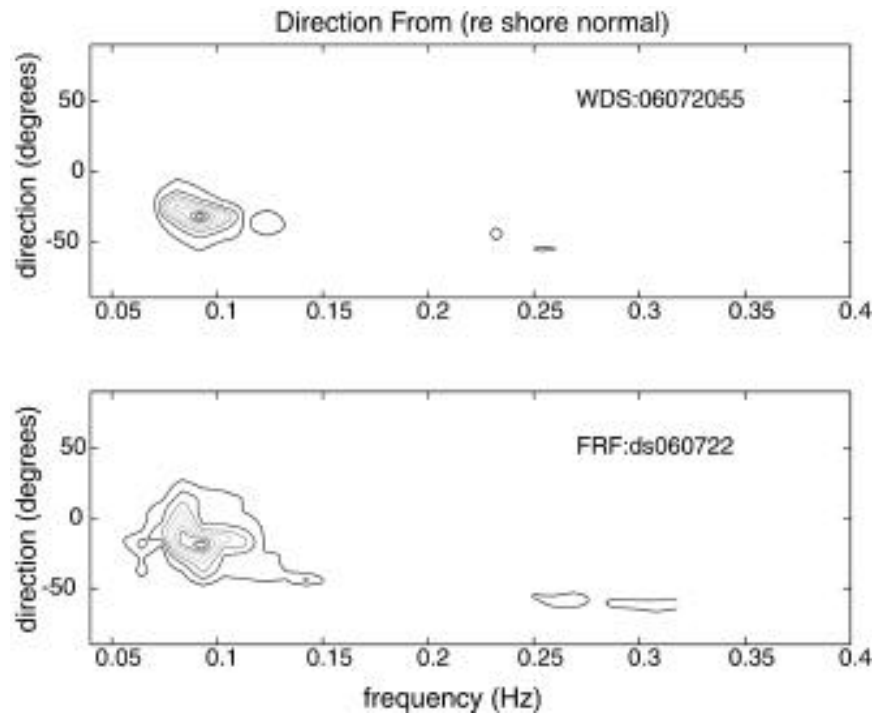

Fig. 10 


\section{SUMMARY AND CONCLUSIONS:}

We have deployed upward-looking, bottom-mounted $1200 \mathrm{kHz}$ ADCPs in depths of 8 meters at FRF, Duck NC, and 11 meters near the SIO pier in La Jolla CA. The principal results obtained to date from these field tests are:

- Wave height and frequency-direction spectra estimated from ADCP velocity measurements compare well to the corresponding spectra computed from a nearby bottom-mounted array of pressure gages.

- Wave parameters such as significant height, peak period, and peak direction also compare well [7].

- Estimates of the wave height spectrum via echoranging to the surface, when available, are in close agreement with estimates derived from the ADCP velocity measurements.

- Specialized beam configurations, such as the $3+1$ geometry, offer a slight improvement in performance over conventional Janus slant-beam ADCPs.

The main topic for further research is to validate this technique in deeper water. This will require the use of lowerfrequency ADCPs having more range, but correspondingly less range resolution. In this case, more sophisticated surface detection schemes than the one employed here likely will be required to measure wave height using echo-ranging, particularly for Janus-configured instruments. However, we expect that the performance of echo-ranging as implemented here for determining water depth on tidal time scales will continue to be adequate.

\section{REFERENCES:}

[1] Brumley, B.H., R.G. Cabrera, K.L. Dienes and E.A. Terray (1983): Performance of a broad-band acoustic Doppler current profiler. J. Oceanic Engr., 16(4), 402-407.

[2] Capon, J. (1969): High-resolution frequency-wavenumber spectrum analysis. Proc. IEEE, 57, 1408-1418; also Corrigendum in Proc. IEEE (Lett.), 59, 112 (1971).
[3] Herbers, T.H.C., R.L. Lowe and R.T. Guza (1991): Field verification of acoustic Doppler surface gravity wave measurements. J. Geophys. Res., 96(9), 17,023-17,035.

[4] Johnson, D.H. and D.E. Dudgeon (1993): Array Signal Processing. Prentice Hall, pp. 533.

[5] Krogstad, H.E., R.L. Gordon and M.C. Miller (1988): High resolution directional wave spectra from horizontallymounted acoustic Doppler current meters. J. Atmos. and Oceanic Technol., 5, 340-352.

[6] Pawka, S.S. (1983): Island shadows in wave directional spectra. J. Geophys. Res., 88, 2579-2591.

[7] Terray, E.A., R.L. Gordon and B.H. Brumley (1997): Measuring wave height and direction using upward-looking ADCPs. Proc. of Oceans'97. Oceans'97 MTS/IEEE, 287290.

[8] Terray, E.A., H.E. Krogstad, R. Cabrera, R.L. Gordon and A. Lohrmann (1990): Measuring wave direction using upward-looking Doppler sonar. Proc. of the IEEE 4th Working Conf. on Current Measurement, G.F. Appell and T.B. Curtin (eds.), IEEE Press, 252-257.

[9] Visbeck, M. and J. Fischer (1995): Sea surface conditions remotely sensed by upward-looking ADCPs. J. Atmos. And Oceanic Technol., 12, 141-149.

[10] Zedel, L. (1994): Deep ocean wave measurements using a vertically oriented sonar. J. Atmos. and Oceanic Technol., 11(1), 182-191.

Acknowledgements: This work was funded under NSF SBIR Grant 9114706 to RD Instruments Inc. Data from their bottommounted pressure gage array were provided by the Field Research Facility (FRF) of the U.S. Army Engineer Waterways Experiment Station's Coastal Engineering Research Center at Duck, NC, and the authors want to thank C. Baron, B. Birkemeyer and C. Long for their assistance, and for permission to use the data from the FRF linear array. The authors also want to acknowledge the contribution of R. Lee Gordon to all phases of this work, and particularly to the echo-ranging technique. 